\title{
Unification of the Bernstein-type polynomials and their applications
}

Yilmaz Simsek ${ }^{*}$

\section{"Correspondence:}

ysimsek@akdeniz.edu.tr

Department of Mathematics,

Faculty of Science, University of

Akdeniz, Antalya, TR-07058, Turkey

\begin{abstract}
In this paper, we investigate some new identities related to the unification of the Bernstein-type polynomials, Bernoulli polynomials, Euler numbers and Stirling numbers of the second kind. We also give some remarks and applications of the Bernstein-type polynomials related to solving high even-order differential equations by using the Bernstein-Galerkin method. We also give some applications on these polynomials and differential equations.

MSC: 11B68; 12D10; 14F10; 26C05; 26C10; 30B40; 30C15; 42A38; 44A10

Keywords: Bernstein polynomials; generating function; Bezier curves; Laplace transform; functional equation; high-order differential equations; Bernstein-Galerkin method; Bernoulli polynomials; Bernoulli numbers; Euler polynomials; Euler numbers; Genocchi polynomials; Genocchi numbers; Stirling numbers of the second kind
\end{abstract}

\section{Introduction}

Generating functions play an important role in the investigation of various useful properties of the sequences and differential equations. These functions are also used to find many properties and formulas for the sequences. In [1], the author constructed certain generating functions for the unification of the classical Bernstein polynomials. Using these generating functions, the author derived several interesting and useful identities for these polynomials. The Bernstein polynomials have been defined by many different ways, for example, by $q$-series, by complex function and by many algorithms. The Bernstein polynomials are used in approximations of functions as well as in other fields such as smoothing in statistics, in numerical analysis, constructing the Bezier curves. The Bernstein polynomials are also used to solve differential equations.

According to Farouki [2], the Bernstein polynomial basis was introduced 100 years ago (Bernstein, 1912) as a means to constructively prove the ability of polynomials to approximate any continuous function, to any desired accuracy, over a prescribed interval. Their slow convergence rate and the lack of digital computers to efficiently construct them caused the Bernstein polynomials to lie dormant in the theory rather than practice of approximation for the better part of a century. The Bernstein coefficients of a polynomial provide valuable insight into its behavior over a given finite interval, yielding many useful properties and elegant algorithms that are now being increasingly adopted in other application domains.

o 2013 Simsek; licensee Springer. This is an Open Access article distributed under the terms of the Creative Commons Attribution License (http://creativecommons.org/licenses/by/2.0), which permits unrestricted use, distribution, and reproduction in any medium, provided the original work is properly cited. 
Recently, the author [1] introduced and investigated the following generating functions which use a unification of the classical Bernstein polynomials:

$$
\mathcal{F}(t, b, s: x)=\frac{2^{b} x^{b s}\left(\frac{t}{2}\right)^{b s} e^{t(1-x)}}{(b s) !}
$$

where $b, s \in \mathbb{N}_{0}:=\{0,1,2,3, \ldots\}, t \in \mathbb{C}$ and $x \in[0,1]$. The following function is a generating function of the polynomials $\mathfrak{S}_{n}(b, s, x)$

$$
\mathcal{F}(t, b, s: x)=\sum_{n=0}^{\infty} \mathfrak{S}_{n}(b, s, x) \frac{t^{n}}{n !}
$$

where $\mathfrak{S}_{0}(b, s, x)=\cdots=\mathfrak{S}_{b s-1}(b, s, x)=0$.

An explicit formula of the polynomials $\mathfrak{S}_{n}(b, s, x)$ is given by the following theorem [1].

Theorem 1.1 Let $x \in[0,1]$. Let $b, n$ and $s$ be nonnegative integers. If $n \geq b s$, then we have

$$
\mathfrak{S}_{n}(b, s, x)=\left(\begin{array}{l}
n \\
b s
\end{array}\right) \frac{x^{b s}(1-x)^{n-b s}}{2^{b(s-1)}}
$$

Remark 1.2 If we set $s=1$ in (2), we have

$$
\mathfrak{S}_{n}(b, 1, x)=B_{b}^{n}(x)
$$

which denotes the classical Bernstein basis function ( $c f$. [1-7]). Consequently, the polynomials $\mathfrak{S}_{n}(b, s, x)$ are a unification of the Bernstein polynomials.

The remainder of this study is organized as follows.

Section 2: We give many properties of the unification of the Bernstein-type polynomials: partition of unity, alternating sum, subdivision property. We also give many functional equations and differential equations of this generating function. Using these equations, many properties of the unification of the Bernstein-type polynomials can be found. Section 3: Integral representations of the unification of the Bernstein-type polynomials are given. Using these representations, we give an identity. Section 4: By using the Laplace transform, we find some identities of the unification of the Bernstein-type polynomials. Section 5: By using a new generating function, we prove the Marsden identity for the unification of the Bernstein-type polynomials. Section 6: By using generating functions, we give relations between the unification of the Bernstein-type polynomial, the unification of the Bernoulli polynomial of higher order and the Stirling numbers of the second kind. Section 7: By using the unification of the Bernstein-type polynomials and the Bernstein-Galerkin methods, we solve high even-order differential equations. Section 8: We give some remarks on the unification of the Bernstein-type polynomials and Beziertype curves.

\section{Properties of the unification of the Bernstein-type polynomials}

In this section, we investigate some properties of the unification of the Bernstein-type polynomials. 


\subsection{Partition of unity}

The unification of the Bernstein-type polynomials $\mathfrak{S}_{n}(b, s, x)$ does not have partition of unity. That is, by using (1), we derive the following functional equation:

$$
\sum_{s=0}^{\infty} \mathcal{F}(t, 1, s: x)=2 e^{\frac{t}{2}(2-x)}
$$

By using the same method as that in [7] and (2), we arrive at the formula for the polynomials $\mathfrak{S}_{n}(1, s, x)$

$$
\sum_{s=0}^{n} \mathfrak{S}_{n}(1, s, x)=2^{1-n}(2-x)^{n}
$$

Remark 2.1 The polynomials $2^{b(s-1)} \mathfrak{S}_{n}(b, s, x)$ have partition of unity. That is,

$$
\sum_{b s=0}^{n} 2^{b(s-1)} \mathfrak{S}_{n}(b, s, x)=1 .
$$

\subsection{Alternating sum}

By using (1), we derive the following functional equation which is used to find an alternating sum of the unification of the Bernstein-type polynomials $\mathfrak{S}_{n}(b, s, x)$ :

$$
\sum_{s=0}^{\infty}(-1)^{s} \mathcal{F}(t, 1, s: x)=2 e^{\frac{t}{2}(2-3 x)}
$$

By using the same method as that in [7] and (2), we arrive at a formula for the alternating sum of the polynomials $\mathfrak{S}_{n}(1, s, x)$, which is given by the following theorem.

\section{Theorem 2.2}

$$
\sum_{s=0}^{n}(-1)^{s} \mathfrak{S}_{n}(1, s, x)=2^{1-n}(2-3 x)^{n}
$$

\subsection{Subdivision property}

Here, we give partial differential equations and a functional equation of the generating function for the unification of the Bernstein-type polynomials $\mathfrak{S}_{n}(b, s, x)$. By using this functional equation, we derive the subdivision property unification of the Bernstein-type polynomials $\mathfrak{S}_{n}(b, s, x)$.

We set

$$
\mathcal{F}(t, b, s: y x)=\mathcal{F}(y t, b, s: x) e^{t(1-y)} .
$$

By using the above functional equation and (2), we derive the subdivision property for the polynomials $\mathfrak{S}_{n}(b, s, x)$ by the following theorem.

\section{Theorem 2.3}

$$
\mathfrak{S}_{n}(b, s, x y)=\sum_{k=b s}^{n} 2^{k-1} \mathfrak{S}_{k}(b, s, x) \mathfrak{S}_{n}(1, k, y)
$$


or

$$
\mathfrak{S}_{n}(b, s, x y)=\sum_{k=b s}^{n} \mathfrak{S}_{k}(b, s, x) B_{k}^{n}(y)
$$

where $B_{k}^{n}(y)$ denotes the classical Bernstein basis function.

Remark 2.4 Substituting $s=1$ into (4), we obtain the subdivision property for the classical Bernstein basis functions:

$$
B_{b}^{n}(x y)=\sum_{k=b}^{n} B_{b}^{k}(x) B_{k}^{n}(y)
$$

Using (3), we give the following partial differential equations:

$$
\frac{\partial}{\partial y} \mathcal{F}(t, b, s: y x)=e^{t(1-y)} \frac{\partial}{\partial y} \mathcal{F}(y t, b, s: x)-t \mathcal{F}(y t, b, s: x)
$$

and

$$
\frac{\partial}{\partial x} \mathcal{F}(t, b, s: y x)=e^{t(1-y)} \frac{\partial}{\partial x} \mathcal{F}(y t, b, s: x)
$$

By applying these partial differential equations, we obtain the following derivative relations which are related to the subdivision property unification of the Bernstein-type polynomials $\mathfrak{S}_{n}(b, s, x)$, respectively:

\section{Theorem 2.5}

$$
\frac{\partial}{\partial y} \mathfrak{S}_{n}(b, s, x y)=\sum_{j=0}^{n} j B_{j}^{n}(y) \mathfrak{S}_{j}(b, s, x)-n y^{n-1} \mathfrak{S}_{n-1}(b, s, x)
$$

and

$$
\frac{\partial}{\partial x} \mathfrak{S}_{n}(b, s, x y)=\sum_{j=0}^{n} B_{j}^{n}(y) \frac{\partial}{\partial x} \mathfrak{S}_{j}(b, s, x) .
$$

\section{Integral representations}

In this section, we derive integral representations of the unification of the Bernstein-type polynomials $\mathfrak{S}_{n}(b, s, x)$. We also give an identity which connects the binomial coefficients, gamma and beta functions.

The beta function $B(\alpha, \beta)$ is a function of two complex variables $\alpha$ and $\beta$, defined by

$$
B(\alpha, \beta)=\int_{0}^{1} t^{\alpha-1}(1-t)^{\beta-1} d t=B(\beta, \alpha) \quad(\Re(\alpha)>0, \Re(\beta)>0)
$$

(cf. [8, p.9, Eq. (60)]). The beta function is related to the gamma function; one has

$$
B(\alpha, \beta)=\frac{\Gamma(\alpha) \Gamma(\beta)}{\Gamma(\alpha+\beta)} .
$$


Replacing $\alpha$ by $n \in \mathbb{Z}^{+}$and $\beta$ by $m \in \mathbb{Z}^{+}$in the above equation, we get

$$
B(\alpha, \beta)=\frac{\Gamma(n) \Gamma(m)}{\Gamma(n+m)}=\frac{(n-1) !(m-1) !}{(n+m-1) !}
$$

(cf. [8, p.9, Eq. (62)]).

\section{Theorem 3.1}

$$
\int_{0}^{1} \mathfrak{S}_{n}(b, s, x) d x=2^{b(1-s)}\left(\begin{array}{l}
n \\
b s
\end{array}\right) B(b s+1, n-b s+1)
$$

or

$$
\int_{0}^{1} \mathfrak{S}_{n}(b, s, x) d x=2^{b(1-s)}\left(\begin{array}{c}
n \\
b s
\end{array}\right) \sum_{l=0}^{n-b s}(-1)^{n-b s-l}\left(\begin{array}{c}
n-b s \\
l
\end{array}\right) \frac{1}{n-l+1} .
$$

Proof

$$
\int_{0}^{1} \mathfrak{S}_{n}(b, s, x) d x=2^{b(1-s)}\left(\begin{array}{c}
n \\
b s
\end{array}\right) \int_{0}^{1} x^{b s}(1-x)^{n-b s} d x,
$$

where

$$
b s \leq n .
$$

By using (5), we easily arrive at the desired result.

Binomial coefficients play an important role in mathematics and mathematical physics, especially in statistics, probability and analytic number theory. Therefore, by using (7) and (8), we derive the following identity related to the binomial coefficients, gamma and beta functions:

$$
\sum_{l=0}^{n-b s}(-1)^{n-b s-l}\left(\begin{array}{c}
n-b s \\
l
\end{array}\right) \frac{1}{n-l+1}=\frac{1}{(n+1)\left(\begin{array}{c}
n \\
b s
\end{array}\right)}
$$

\section{Identities}

In this section, by using the Laplace transform, we give some identities of the unification of the Bernstein-type polynomials $\mathfrak{S}_{n}(b, s, x)$.

Using the generating function in (2), we get

$$
e^{-t} \sum_{n=0}^{\infty} \mathfrak{S}_{n}(b s, x) \frac{t^{n}}{n !}=\frac{2^{b} x^{b s}\left(\frac{t}{2}\right)^{b s}}{(b s) !} e^{-x t} .
$$

Integrating equation (10) (by parts) with respect to $t$ from zero to infinity, we have

$$
\sum_{n=0}^{\infty} \frac{\mathfrak{S}_{n}(b, s, x)}{n !} \int_{0}^{\infty} e^{-t} t^{n} d t=\frac{2^{b(1-s)} x^{b s}}{(b s) !} \int_{0}^{\infty} t^{b s} e^{-x t} d t
$$


If we appropriately use the case $x>0$ of the following Laplace transform of the function $f(t)=t^{k}$ :

$$
\int_{0}^{\infty} t^{k} e^{-u t} d t=\frac{k !}{u^{k+1}}
$$

by substituting (12) into (11), we arrive at the following theorem.

Theorem 4.1 Let $|1-x|<1$. Then we have

$$
\sum_{n=0}^{\infty} x \mathfrak{S}_{n}(b, s, x)=2^{b(1-s)} .
$$

Remark 4.2 If we set $s=1$ in (13), then we arrive at Theorem 15 in [7].

We modify (2) as follows:

$$
e^{x t} \sum_{n=0}^{\infty} \mathfrak{S}_{n}(b, s, x) \frac{t^{n}}{n !}=\frac{2^{b} x^{b s}\left(\frac{t}{2}\right)^{b s}}{(b s) !} e^{t} .
$$

From the above equation, we get

$$
\sum_{n=0}^{\infty} x^{n} \frac{t^{n}}{n !} \sum_{n=0}^{\infty} \mathfrak{S}_{n}(b, s, x) \frac{t^{n}}{n !}=\frac{2^{b(1-s)} x^{b s}}{(b s) !} \sum_{n=0}^{\infty} \frac{t^{n+b s}}{n !} .
$$

Therefore, we arrive at the following theorem.

\section{Theorem 4.3}

$$
\sum_{j=0}^{n}\left(\begin{array}{l}
n \\
j
\end{array}\right) x^{j} \mathfrak{S}_{n-j}(b, s, x)=\left(\begin{array}{c}
n \\
b s
\end{array}\right) 2^{b(1-s)} x^{b s} .
$$

\section{Marsden identity}

In this section, by using generating functions, we prove the Marsden identity for the unification of the Bernstein-type polynomials $\mathfrak{S}_{n}(b, s, x)$. This identity is associated with a formula for rational linear transformation of $B$-splines, which are of interest in computeraided geometric design and approximation theory.

We set

$$
h(x, u, t)=\sum_{n=0}^{\infty}(x-u)^{n} \frac{t^{n}}{n !}
$$

or

$$
h(x, u, t)=e^{(x-u) t} .
$$

We derive the following functional equation:

$$
h(x, u, t)=h(1, u, x t) h(x, 1, u t) .
$$


From the above functional equation, we get

$$
\sum_{n=0}^{\infty}(x-u)^{n} \frac{t^{n}}{n !}=\left(\sum_{n=0}^{\infty}(1-u)^{n} \frac{x^{n} t^{n}}{n !}\right)\left(\sum_{n=0}^{\infty}(-1)^{n}(1-x)^{n} \frac{u^{n} t^{n}}{n !}\right) .
$$

Therefore

$$
\sum_{n=0}^{\infty}(x-u)^{n} \frac{t^{n}}{n !}=\sum_{n=0}^{\infty}\left(\sum_{j=0}^{n}(-1)^{n-j}\left(\begin{array}{l}
n \\
j
\end{array}\right) x^{j}(1-x)^{n-j} u^{n-j}(1-u)^{j}\right) \frac{t^{n}}{n !} .
$$

From the above equation, we have

$$
\sum_{n=0}^{\infty}(x-u)^{n} \frac{t^{n}}{n !}=\sum_{n=0}^{\infty}\left(\sum_{j=0}^{n}(-1)^{n-j} \frac{2^{n-2}}{\left(\begin{array}{c}
n \\
n-j
\end{array}\right)} \mathfrak{S}_{n}(1, j, x) \mathfrak{S}_{n}(1, n-j, u)\right) \frac{t^{n}}{n !} .
$$

By comparing the coefficients of $\frac{t^{n}}{n !}$ on the both sides of the above equation, we obtain

$$
(x-u)^{n} \frac{1}{n !}=\frac{1}{n !} \sum_{j=0}^{n}(-1)^{n-j} \frac{2^{n-2}}{\left(\begin{array}{c}
n \\
n-j
\end{array}\right)} \mathfrak{S}_{n}(1, j, x) \mathfrak{S}_{n}(1, n-j, u) .
$$

Therefore, we arrive at the Marsden identity which is given by the following theorem.

\section{Theorem 5.1}

$$
(x-u)^{n}=\sum_{j=0}^{n}(-1)^{n-j} \frac{2^{n-2}}{\left(\begin{array}{c}
n \\
n-j
\end{array}\right)} \mathfrak{S}_{n}(1, j, x) \mathfrak{S}_{n}(1, n-j, u) .
$$

Remark 5.2 By using (14), we also obtain the Marsden identity for the classical Bernstein polynomials $B_{j}^{n}(x)$ as follows:

$$
(x-u)^{n}=\sum_{j=0}^{n} \frac{(-1)^{n-j}}{\left(\begin{array}{c}
n \\
n-j
\end{array}\right)} B_{j}^{n}(x) B_{n-j}^{n}(u) .
$$

\section{Relations between the polynomial $\mathfrak{S}_{n}(b, s, x)$, unification of the Bernoulli polynomial of higher order and Stirling numbers of the second kind}

The so-called unification of the Bernoulli, Euler and Genocchi polynomials $\mathcal{Y}_{n, \beta}(x ; k, a, b)$ were defined by Ozden [9]. The polynomials $\mathcal{Y}_{n, \beta}(x ; k, a, b)$ are defined by means of the following generating function:

$$
\mathfrak{f}_{a, b}(x ; t ; k, \beta)=\frac{2^{1-k} t^{k} e^{t x}}{\beta^{b} e^{t}-a^{b}}=\sum_{n=0}^{\infty} \mathcal{Y}_{n, \beta}(x ; k, a, b) \frac{t^{n}}{n !},
$$

where $k$ is an integer parameter, $a$ and $b$ are real parameters and $\beta$ is a complex parameter. Observe that

$$
\mathcal{Y}_{n, \beta}(0 ; k, a, b)=\mathcal{Y}_{n, \beta}(k, a, b)
$$

(cf. $[9,10])$.

The above generating function is related to some special polynomials as follows. 
Remark 6.1 Substituting $a=b=k=1$ into (15), we have the Apostol-Bernoulli polynomials $(c f .[11-13])$ :

$$
\mathcal{Y}_{n, \beta}(x ; 1,1,1)=\mathcal{B}_{n}(x, \beta) ;
$$

substituting $b=1, k=0$ and $a=-1$ into (15), we have the Apostol-Euler polynomials:

$$
\mathcal{Y}_{n, \beta}(x ; 0,-1,1)=\mathcal{E}_{n}(x, \beta) ;
$$

substituting $b=1, k=1$ and $a=-1$ into (15), we have the Apostol-Genocchi polynomials:

$$
\mathcal{Y}_{n, \beta}(x ; 1,-1,1)=\frac{1}{2} \mathcal{G}_{n}(x, \beta) ;
$$

substituting $\beta=b=k=a=1$ into (15), we have

$$
\mathcal{Y}_{n, 1}(x ; 1,1,1)=B_{n}(x),
$$

where $B_{n}(x)$ denotes the classical Bernoulli polynomials and substituting $\beta=b=k=1$ and $a=-1$ into (15), we have

$$
\mathcal{Y}_{n, 1}(x ; 0,-1,1)=E_{n}(x),
$$

where $E_{n}(x)$ denotes the classical Euler polynomials.

Now, the modification of (15) is given by

$$
\frac{\left(\frac{2}{a}\right)^{v}\left(\frac{t}{2}\right)^{k v} e^{t x}}{\left(\frac{\beta}{a} e^{t}-1\right)^{v}}=\sum_{n=0}^{\infty} \mathcal{Y}_{n, \frac{\beta}{a}}^{(v)}(x ; k, 1,1) \frac{t^{n}}{n !} .
$$

The following definition provides a natural generalization and unification of $\lambda$-Stirling numbers of the second kind, which is defined by Srivastava [12, 13].

Definition 6.2 Let $\lambda \in \mathbb{C}$ and $v \in \mathbb{N}_{0}$. The generalized $\lambda$-Stirling type numbers of the second kind $\mathcal{S}(n, v ; \lambda)$ are defined by means of the following generating function:

$$
f_{S, v}(t ; \lambda)=\frac{\left(\lambda e^{t}-1\right)^{v}}{v !}=\sum_{n=0}^{\infty} \mathcal{S}(n, v ; \lambda) \frac{t^{n}}{n !} .
$$

Remark 6.3 By setting $\lambda=1$ in (17), we get

$$
S(n, v)=\mathcal{S}(n, v ; 1),
$$

where $S(n, v)$ denotes the Stirling numbers of the second kind. It is also well known that

$$
x^{n}=\sum_{v=0}^{n}\left(\begin{array}{l}
x \\
v
\end{array}\right) v ! S(n, v)
$$


so that

$$
S(n, 0)=\delta_{n, 0}, \quad S(n, 1)=S(n, n)=1 \quad \text { and } \quad S(n, n-1)=\left(\begin{array}{l}
n \\
2
\end{array}\right),
$$

$\delta_{n, 0}$ being the Kronecker symbol $(c f .[8,12,14,15])$.

Theorem 6.4 Let $b, n$ and $s$ be nonnegative integers with $n \geq b s$. Then we have

$$
2^{b(1-s)} a^{-b}(1-x)^{n-b s}=\sum_{j=b s}^{n} \frac{\left(\begin{array}{c}
n \\
j
\end{array}\right)}{\left(\begin{array}{c}
n \\
b s
\end{array}\right)} \mathcal{S}\left(j, b s ; \frac{\beta}{a}\right) \mathcal{Y}_{n-j, \frac{\beta}{a}}^{(b s)}(1-x ; k, 1,1),
$$

where $\mathcal{Y}_{n, \frac{\beta}{a}}^{(v)}(x ; k, 1,1)$ and $\mathcal{S}\left(j, b s ; \frac{\beta}{a}\right)$ denote the unification Bernoulli polynomial of higher order and $\frac{\beta}{a}$-Stirling numbers of the second kind, respectively.

Proof By (2), we have

$$
a^{b} x^{b s}\left(\frac{\left(\frac{\beta}{a} e^{t}-1\right)^{b s}}{(b s) !}\right)\left(\frac{\left(\frac{2}{a}\right)^{b}\left(\frac{t}{2}\right)^{b s} e^{(1-x) t}}{\left(\frac{\beta}{a} e^{t}-1\right)^{b s}}\right)=\sum_{n=0}^{\infty} \mathfrak{S}_{n}(b, s, x) \frac{t^{n}}{n !} .
$$

By using (2), (16) and (17) in the above equation, we have

$$
\sum_{n=0}^{\infty} \mathfrak{S}_{n}(b s, x) \frac{t^{n}}{n !}=a^{b} x^{b s}\left(\sum_{n=0}^{\infty} \mathcal{Y}_{n, \frac{\beta}{a}}^{(b s)}(1-x ; k, 1, e)\right)\left(\sum_{n=0}^{\infty} \mathcal{S}\left(n, b s ; \frac{\beta}{a}\right) \frac{t^{n}}{n !}\right)
$$

From the above equation, after some calculation, we find the desired result.

Theorem 6.5 Let $b$ and $n$ be nonnegative integers with $n \geq b$. Then we have

$$
(1-x)^{n}=\sum_{m=0}^{n} \sum_{j=0}^{b}\left(\begin{array}{l}
n \\
m
\end{array}\right)\left(\begin{array}{l}
b \\
j
\end{array}\right) j^{m} E_{n-m}^{(b)}(1-x)
$$

where $E_{n-m}^{(b)}(1-x)$ denotes the Euler polynomials of higher order.

Proof By (2), we have

$$
2^{b} e^{(1-x) t}\left(\frac{e^{t}+1}{e^{t}+1}\right)^{b}=\sum_{n=0}^{\infty} \mathfrak{S}_{n}(b, 0, x) \frac{t^{n}}{n !} .
$$

From the above, we have

$$
\begin{aligned}
\sum_{n=0}^{\infty} \mathfrak{S}_{n}(b, 0, x) \frac{t^{n}}{n !} & =e^{(1-x) t}\left(\frac{2}{e^{t}+1}\right)^{b}\left(e^{t}+1\right)^{b} \\
& =\left(\sum_{n=0}^{\infty} \sum_{j=0}^{b}\left(\begin{array}{l}
b \\
j
\end{array}\right) j^{n} \frac{t^{n}}{n !}\right)\left(\sum_{n=0}^{\infty} E_{n}^{(b)}(1-x) \frac{t^{n}}{n !}\right) .
\end{aligned}
$$


By using the Cauchy product in the above, after some calculation, we find the desired result.

We recall from the work of Gould [14, Vol. 7, Eq. (2.45)] that

$$
\sum_{j=0}^{b}\left(\begin{array}{l}
b \\
j
\end{array}\right) j^{m}=2^{b} \sum_{j=0}^{m} \frac{1}{2^{j}}\left(\begin{array}{l}
b \\
j
\end{array}\right) B_{j, j}^{m}
$$

where

$$
B_{j, j}^{m}=j ! S(m, j)
$$

By substituting (20) into (19), we arrive at the following result.

Corollary 6.6 The following identity holds true:

$$
\mathfrak{S}_{n}(b, 0, x)=\sum_{m=0}^{n} \sum_{j=0}^{m}\left(\begin{array}{l}
n \\
m
\end{array}\right)\left(\begin{array}{l}
b \\
j
\end{array}\right) 2^{b-j} E_{n-m}^{(b)}(1-x) B_{j, j}^{m} .
$$

\section{Unification of the Bernstein-type polynomials for solving high even-order differential equations by the Bernstein-Galerkin methods}

In [4], Doha et al. gave an application of the Bernstein polynomials for solving high evenorder differential equations by using the Bernstein-Galerkin and the Bernstein-PetrovGalerkin methods. The methods do not contain generating functions for proving explicitly the derivatives formula of the Bernstein polynomials of any degree and for any order in terms of Bernstein polynomials themselves. Here, we prove this formula for the unification of the Bernstein-type polynomials $\mathfrak{S}_{n}(b, s, x)$ by a higher-order partial differential equation and functional equations. We also give some remarks and applications related to these polynomials and the Bernstein-Galerkin method.

We modify (1) as follows:

$$
\begin{aligned}
\mathcal{F}(t, b-k, s-k: x) & =\frac{2^{b-k} x^{b s-k}\left(\frac{t}{2}\right)^{b s-k} e^{t(1-x)}}{(b s-k) !} \\
& =\sum_{n=0}^{\infty} \mathfrak{S}_{n}(b-k, s-k, x) \frac{t^{n}}{n !},
\end{aligned}
$$

where $k \in \mathbb{N}_{0}=\{0,1,2, \ldots\}$ and $x \in[0,1]$. Let $b, k, n$ and $s$ be nonnegative integers and $n \geq b s-k \geq 0$, then we get

$$
\mathfrak{S}_{n}(b-k, s-k, x)=\left(\begin{array}{c}
n \\
b s-k
\end{array}\right) \frac{x^{b s-k}(1-x)^{n-b s+k}}{2^{b(s-1)}},
$$

so that, obviously,

$$
\mathfrak{S}_{n}(b, s, x)=\mathfrak{S}_{n}(b-0, s-0, x) .
$$

By using the same method as in [15], we now give a higher-order partial differential equation for the generating function $\mathcal{F}(t, b, s: x)$ as follows. 
We set

$$
g(t, x ; b, s)=\frac{2^{b} x^{b s}\left(\frac{t}{2}\right)^{b s}}{(b s) !}
$$

and

$$
h(t, x)=e^{t(1-x)} .
$$

We have

$$
\mathcal{F}(t, b, s: x)=g(t, x ; b, s) h(t, x) .
$$

By using Leibnitz's formula for the $v$ th derivative, with respect to $x$, of the product $\mathcal{F}(t, b, s: x)$ of the above two functions, we obtain the following higher-order partial differential equation:

$$
\frac{\partial^{v} \mathcal{F}(t, b, s: x)}{\partial x^{v}}=\sum_{j=0}^{v}\left(\begin{array}{l}
v \\
j
\end{array}\right)\left(\frac{\partial^{j} g(t, x ; b, s)}{\partial x^{j}}\right)\left(\frac{\partial^{v-j} h(t, x)}{\partial x^{v-j}}\right) .
$$

By using (1) in the above partial differential equation, we get the following higher order partial differential equation:

$$
\frac{\partial^{v} \mathcal{F}(t, b, s: x)}{\partial x^{v}}=\sum_{j=0}^{v}(-1)^{v-j}\left(\begin{array}{l}
v \\
j
\end{array}\right) \mathcal{F}(t, b-j, s-j: x)
$$

By substituting (21) into the above equation, after some calculation, we arrive at the following theorem.

Theorem 7.1 Let $x \in[a, b]$. Let $b$, s and $v$ be nonnegative integers with $n \geq b s$. Then we have

$$
\mathfrak{S}_{n}^{(v)}(b, s, x)=\frac{n !}{(n-v) !} \sum_{j=0}^{v}(-1)^{v-j}\left(\begin{array}{l}
v \\
j
\end{array}\right) \mathfrak{S}_{n-v}(b-j, s-j, x),
$$

where

$$
\mathfrak{S}_{n}^{(v)}(b, s, x)=\frac{d^{v} \mathfrak{S}_{n}(b, s, x)}{d x^{v}} .
$$

Integrating equation (22) (by parts) with respect to $x$ from 0 to 1 and using Theorem 3.1, we have

$$
\int_{0}^{1} \mathfrak{S}_{n}(b, s, x) d x=\frac{2^{b(1-s)}}{n+1},
$$

for all $b$ and $s$.

$$
\int_{0}^{1} \mathfrak{S}_{n}^{(v)}(b, s, x) \mathfrak{S}_{n}(b, s, x) d x=\frac{2^{2 b(1-s)} n !}{(2 n+1)(n-v) !}\left(\begin{array}{c}
n \\
b s
\end{array}\right) \sum_{j=0}^{v}(-1)^{\nu-j} \frac{\left(\begin{array}{l}
v \\
j
\end{array}\right)\left(\begin{array}{c}
n \\
b s-j
\end{array}\right)}{\left(\begin{array}{c}
2 n \\
2 b s-j
\end{array}\right)} .
$$


We recall from the work of Doha et al. [4] that if $f(x)$ is a differentiable function of degree $m$ and defined on $[0,1]$, then a linear combination of the Bernstein polynomials can be written. Therefore, we can easily have

$$
f(x)=\sum_{b, s=0}^{m} c_{b, s, m} \mathfrak{S}_{m}(b, s, x)
$$

where $b s \leq m$, otherwise $\mathfrak{S}_{m}(b, s, x)=0$ and

$$
\sum_{b, s=0}^{m}=\sum_{b=0}^{m} \sum_{s=0}^{m}
$$

By using the same method as in [4], we write

$$
f^{(v)}(x)=\frac{d^{v} f(x)}{d x^{v}}=\sum_{b, s=0}^{m} c_{b, s, m} \frac{d^{v} \mathfrak{S}_{m}(b, s, x)}{d x^{v}}
$$

where $b s \leq m$, otherwise $\mathfrak{S}_{m}(b, s, x)=0$. We now give an application for the solution of high even-order differential equations. We also recall from the work of Doha et al. [4] that for $x \in[0,1]$,

$$
f(x)=u^{(2 m)}+\sum_{j=1}^{2 m-1} \gamma_{j} u^{(j)}+\gamma_{0} u
$$

by the following boundary conditions:

$$
u^{(v)}(0)=0, \quad u^{(v)}(1)=0 ; \quad 0 \leq v \leq m-1
$$

(cf. [4]). By using the same method as that of Doha et al. [4], we apply the unification of the Bernstein-type polynomials $\mathfrak{S}_{m}(b, s, x)$ to the Bernstein-Galerkin approximation for solving (24); that is,

$$
Y_{m}=\left\{\mathfrak{S}_{m}(b, s, x): m \geq b s\right\}
$$

and

$$
Z_{m}=\left\{u \in Y_{m}: u^{(v)}(0)=0, u^{(v)}(1)=0 ; 0 \leq v \leq m-1\right\} .
$$

By applying the Bernstein-Galerkin approximation (24), we find $u_{m} \in Z_{m}$ as follows. For solving this equation, we need the following notations, which we recall from the work of Doha et al. [4, p.9, Eq. (4.4)].

The inner product $\langle u, v\rangle$ on $L^{2}(I)$ is defined by

$$
\langle u, v\rangle=\int_{I} u(x) v(x) d x
$$


By using this inner product, we modify (24) as follows:

$$
\left\langle f(x), \mathfrak{g}_{n}(k, x)\right\rangle=\left\langle u_{n}^{(2 m)}, \mathfrak{g}_{n}(k, x)\right\rangle+\sum_{j=1}^{2 m-1} \gamma_{j}\left\langle u_{n}^{(j)}, \mathfrak{g}_{n}(k, x)\right\rangle+\gamma_{0}\left\langle u_{n}, \mathfrak{g}_{n}(k, x)\right\rangle
$$

where $m \leq k \leq n-m, 2 m \leq n$ and

$$
\mathfrak{g}_{n}(k, x)=\mathfrak{S}_{n}(b+k, s+k, x)=\left(\begin{array}{c}
n \\
b s+k
\end{array}\right) \frac{x^{b s+k}(1-x)^{n-b s-k}}{2^{b(s-1)}} .
$$

The matrix representation of the above equation is given by

$$
F=\left(A+\sum_{j=1}^{2 m-1} \gamma_{j} B_{j}+\gamma_{0} B_{0}\right) C
$$

where

$$
\begin{aligned}
& F=\left(f_{m}, f_{m+1}, \ldots, f_{n-m}\right)^{T} ; \quad f_{k}=\left\langle f(x), \mathfrak{g}_{n}(k, x)\right\rangle, \\
& u_{n}(x)=\sum_{k=m}^{n-m} a_{k} \mathfrak{g}_{n}(k, x), \\
& C=\left(a_{m}, a_{m+1}, \ldots, a_{n-m}\right)^{T}, \quad A=\left(a_{k j}\right), \quad B_{j}=\left(b_{k j}^{i}\right), \quad m \leq k, j \leq n-m .
\end{aligned}
$$

By using (23), one can easily find $A, B_{j}(j=0,1,2, \ldots, 2 m-1)$; that is,

$$
a_{k j}=\left\langle\mathfrak{g}_{n}^{(2 m)}(j, x), \mathfrak{g}_{n}(k, x)\right\rangle=\int_{0}^{1} \mathfrak{g}_{n}^{(2 m)}(j, x) \mathfrak{g}_{n}(k, x) d x
$$

and

$$
b_{k j}^{i}=\left\langle\mathfrak{g}_{n}^{(i)}(j, x), \mathfrak{g}_{n}(k, x)\right\rangle=\int_{0}^{1} \mathfrak{g}_{n}^{(i)}(j, x) \mathfrak{g}_{n}(k, x) d x
$$

Remark 7.2 According to Doha et al. [4], it is important to apply the Galerkin-spectral Bernstein approximation for how to choose an appropriate basis for $Z_{m}$ such that the linear system resulting in the Bernstein-Galerkin approximation to (25) is possible. That is,

$$
Z_{m}=\operatorname{span}\left\{\mathfrak{g}_{n}(m, x), \mathfrak{g}_{n}(m+1, x), \ldots, \mathfrak{g}_{n}(n-m, x)\right\}
$$

where $\mathfrak{g}_{n}(k, x) \in Z_{m}$ for all $m \leq k \leq n-m$. The $2 m$ boundary conditions lead to the first $m$, and the least $m$ expansion coefficients are zero.

Remark 7.3 By using the Bernstein-Galerkin and the Bernstein-Petrov-Galerkin methods, Doha et al. [4] solved the following boundary value problem:

$$
u^{(2)}(x)-u(x)=\left(4-2 x^{2}\right) \sin x+4 x \cos x, \quad x \in[0,1]
$$


subject to the boundary conditions $u(0)=u(1)=0$, with the exact solution

$$
u(x)=\left(1-x^{2}\right) \sin x
$$

(cf. see for detail $[4,16])$.

\section{Further remarks on Bezier curves}

The unification of the Bernstein-type polynomials is used to construct Bezier-type curves which are used in computer-aided graphics design and related fields and also in the time domain, particularly in animation and interface design ( $c f .[2,6])$.

The Bezier-type curve of degree $n$ can be generalized by the author [1] as follows:

$$
B_{n}(b, s, x)=\sum_{0 \leq b, s \leq n(b s \leq n)} P_{b, s} \mathfrak{S}_{n}(b, s, x)
$$

where $x \in[0,1], \mathfrak{S}_{n}(b, s, x)$ denotes the unification of the Bernstein-type polynomials and $P_{b, s}$ are the control points.

The unification of the Bernstein-type polynomials might affect the shape of the curves.

Competing interests

The author declares that he has no competing interests.

\section{Author's contributions}

The author completed the paper himself. The author read and approved the final manuscript.

\section{Acknowledgements}

Dedicated to Professor Hari M Srivastava.

The present investigation was supported by the Scientific Research Project Administration of Akdeniz University.

Received: 12 November 2012 Accepted: 27 February 2013 Published: 20 March 2013

\section{References}

1. Simsek, Y: Construction a new generating function of Bernstein-type polynomials. Appl. Math. Comput. 218, 1072-1076 (2011)

2. Farouki, RT: The Bernstein polynomials basis: a centennial retrospective. Comput. Aided Geom. Des. 29, 379-419 (2012)

3. Bernstein, SN: Démonstration du théorème de Weierstrass fondée sur la calcul des probabilités. Commun. Soc. Math. Kharkov, Sér. 2 13, 1-2 (1912-1913)

4. Doha, EH, Bhrawy, AH, Saker, MA: On the derivatives of Bernstein polynomials: an application for the solution of high even-order differential equations. Bound. Value Probl. 2011, Article ID 829543 (2011). doi:10.1155/2011/829543

5. Phillips, GM: Interpolation and Approximation by Polynomials. CMS Books in Mathematics/Ouvrages de Mathématiques de la SMC, vol. 14. Springer, New York (2003)

6. Simsek, Y: Generating functions for the Bernstein-type polynomials: a new approach to deriving identities and applications for these polynomials. Hacet. J. Math. Stat. (in press)

7. Simsek, Y: Functional equations from generating functions: a novel approach to deriving identities for the Bernstein basis functions. Fixed Point Theory Appl. 2013, 80 (2013)

8. Srivastava, HM, Choi, J: Series Associated with the Zeta and Related Functions. Kluwer Academic, Dordrecht (2001)

9. Ozden, $\mathrm{H}$ : Unification of generating function of the Bernoulli, Euler and Genocchi numbers and polynomials. AIP Conf. Proc. 1281, 1125-1128 (2010)

10. Ozden, H, Simsek, Y, Srivastava, HM: A unified presentation of the generating functions of the generalized Bernoulli, Euler and Genocchi polynomials. Comput. Math. Appl. 60, 2779-2787 (2010)

11. Kim, YH, Kim, W, Jang, LC: On the q-extension of Apostol-Euler numbers and polynomials. Abstr. Appl. Anal. 2008, Article ID 296159 (2008)

12. Luo, QM, Srivastava, HM: Some generalizations of the Apostol-Genocchi polynomials and the Stirling numbers of the second kind. Appl. Math. Comput. 217, 5702-5728 (2011)

13. Srivastava, HM: Some generalizations and basic (or q-) extensions of the Bernoulli, Euler and Genocchi polynomials. Appl. Math. Inf. Sci. 5, 390-444 (2011)

14. Gould, HW: http://www.math.wvu.edu/ gould/Vol.7.PDF

15. Simsek, Y: On q-deformed Stirling numbers. Int. J. Math. Comput. 15, 70-80 (2012)

16. Ramadan, MA, Lashien, IF, Zahra, WK: High order accuracy nonpolynomial spline solutions for $2 \mu$ th order two point boundary value problems. Appl. Math. Comput. 204, 920-927 (2008) 
doi:10.1186/1687-2770-2013-56

Cite this article as: Simsek: Unification of the Bernstein-type polynomials and their applications. Boundary Value Problems 2013 2013:56.

Submit your manuscript to a SpringerOpen ${ }^{\circ}$ journal and benefit from:

- Convenient online submission

- Rigorous peer review

- Immediate publication on acceptance

- Open access: articles freely available online

- High visibility within the field

- Retaining the copyright to your article

Submit your next manuscript at $\gg$ springeropen.com 